\title{
New testing software for quantifying discrimination capacity in subjects with ocular pathologies
}

\author{
José J. Castro, José R. Jiménez, Carolina Ortiz, Aixa Alarcón, and Rosario G. Anera \\ University of Granada, Department of Optics, Laboratory of Vision Sciences and Applications, Granada 18071, Spain
}

\begin{abstract}
We develop a new visual test, designed as software for quantifying discrimination capacity under lowillumination conditions. This is an important task in the presence of visual disturbances, such as those perceived by subjects with some ocular pathologies. For this purpose, we propose a visual-disturbance index, checking the test with two groups of observers having different ocular pathologies: a group with unilateral keratitis and another group affected with age-related macular degeneration (ARMD). To compare the test results to objective data, we use a double-pass device to measure the Strehl ratio, a parameter that quantifies the retinal-image quality, taking into account aberrations, retinal reflection, and intraocular scattering working jointly. Diseased eyes present higher disturbance indexes and a lower Strehl ratio compared to their healthy fellow eyes, registering a significant descending correlation between the disturbance index and the Strehl ratio. The lower the Strehl ratio is, the higher the disturbance index for the eyes studied. Therefore, in keratitis and ARMD eyes, our results demonstrate a deterioration in the retinal-image quality and a lower discrimination capacity to peripheral stimuli, reducing visual performance. The test presented here could be useful for the study and time course in different eye diseases, especially those involving an increase in scattered light or alterations in the ocular media, as shown in this work. (C) 2011 Society of Photo-Optical Instrumentation Engineers (SPIE). [DOI: 10.1117/1.3526702]
\end{abstract}

Keywords: discrimination capacity; disturbance index; retinal-image quality; Strehl ratio; keratitis; age-related macular degeneration. Paper 10258R received May 12, 2010; revised manuscript received Oct. 19, 2010; accepted for publication Nov. 12, 2010; published online Jan. 11, 2011.

\section{Introduction}

In the last decade, the use and development of objective optical devices, such as aberrometers and double-pass devices, have expanded to evaluate the optical quality of the human eye. ${ }^{1-5}$ With these devices, the aim is to achieve an objective evaluation of the observer's visual state, spending the least time possible in taking the data. This time savings is a great advantage in clinics, where time spent per patient is limited; also, for older patients, speed of examination avoids unnecessary fatigue in the observer. However, these optical devices were previously large and also costly, sometimes prohibitively so. Without these devices, the visual examination of the subject would be limited to the usual psychophysical tests as well as the study of the refractive state of the subject. Therefore, other subjective tests are needed that require the cooperation of the patient to characterize the visual performance of the subject. The discrimination capacity of the subject is an important visual function for this purpose, allowing us to evaluate the real state of vision of the subject and providing an easy tool for detecting potential visual dysfunctions. For these reasons, a new visual test for quantifying discrimination capacity was developed that needs no specific hardware, is free of charge, and is becoming an accessible and easy tool for clinical applications compared to former complex optical devices. On the other hand, the development of simple visual tests that evaluate visual function is an important task for the study, monitoring, and early diagnosis of different visual pathologies that develop asymptomatically to advanced stages of pathology, such as age-related macular

Address all correspondence to: José J. Castro, University of Granada, Department of Optics, Edificio Mecenas, Facultad de Ciencias, Avenida de Fuentenueva, s/n Granada, Granada 18071, Spain. Tel: 0034958241902; E-mail: jjcastro@ugr.es. degeneration (ARMD), and that could go unnoticed if not adequately tested. In this study, we check the visual test developed for quantifying discrimination capacity in subjects with different ocular pathologies and seek to determine whether these ocular pathologies, which affect the retinal-image quality, cause any deterioration in the discrimination capacity and, consequently, in visual performance.

Basically, the test consists of the discrimination of luminous peripheral stimuli around a more luminous central one. The central luminous stimulus causes a certain amount of intraocular scattering and retinal reflection in the patient's eye, depending on the state of the retina and ocular media of the subject. This effect increases for different ocular pathologies, provoking visual disturbances (such as a veil of stray light over the retinal image, glare, and visual halos perceived by the observer). Using the new visual test developed, performed with software and executed with a PC, we measured the discrimination capacity of the subject and checked two types of patients presenting loss in visual quality, such as keratitis and ARMD patients. One test-selection criterion was that the test should present easy tasks to be performed by patients having different pathologies and ranging widely in age, especially for older patients who are not recommended for complex visual tests. Data compiled with this psychophysical test have been compared to objective data from an optical device (OQAS ${ }^{\mathrm{TM}}$, Optical Quality Analysis System, Visiometrics SL, Tarrasa, Spain) based on the double-pass technique. 


\section{Methods: Test Design}

\subsection{Background}

For the characterization of visual performance, it is important to quantify the discrimination capacity, which deteriorates in the presence of visual disturbances perceived by the observer, especially in subjects having some ocular pathology. A deterioration in the optical quality diminishes the discrimination capacity of the subject, as demonstrated in cataract and refractive surgery patients. ${ }^{6,7}$ The use of devices to evaluate optical quality is not viable in some cases due to the high cost of the instrument or the physical space required, and therefore, simple visual tests are preferable to evaluate visual performance and check the real state of vision of the subject examined. To improve the study of the visual performance as well as evaluate an important visual function - the discrimination capacity — we seek to quantify the visual disturbances perceived by the patients, a key aspect in evaluating visual performance. For this, we developed a new visual test, which is a freeware program, called Halo v1.0 software. This test is based on an experimental device (called a halometer) that was successfully tested for refractive-surgery patients, ${ }^{6}$ although this device showed several limitations. Peripheral stimuli as well as the central one were generated by light-emitting diodes fixed at specific distances. ${ }^{6}$ This characteristic does not allow size changes of the stimuli (neither peripheral nor central), a very important aspect, because the stimulus size needs to be changed for different visual pathologies (the spatial perception of a young subject operated on with LASIK is not the same as that of an older subject operated on for cataracts or suffering ARMD).

In the new test, designed as software, we are able to change the size of the stimuli (peripheral or central) as well as the color, enabling the luminance of the stimulus being modified. All parameters can be modified, depending on the type of patient, the test distance, or other experimental conditions. In this manner, it is possible to customize the test for a particular case or a group of patients to be studied. In addition, this software is a simple test, with no need of additional hardware or physical devices, and could be installed in any computer with minimum requirements, assuring universal use. Another important feature is that the Halo software is a freeware program (i.e., free of charge) therefore promoting the use and expansion of freeware software in clinical applications, a live topic nowadays, and facilitating the use of the visual test in any clinic or research center related to human vision. This test can be an easy and highly useful tool in clinical applications to quantify visual disturbances perceived by the observer as well as to evaluate visual performance. The software can be downloaded from http://www.ugr.es/ labvisgr/, the web page of the Laboratory of Vision Sciences and Applications, University of Granada, Spain. No registration is needed.

\subsection{Description}

As indicated in Sect. 1, the test consists of the discrimination of luminous peripheral stimuli around a central one. In the test, the subject is shown a central high-luminance stimulus over a dark background and, progressively, peripheral stimuli are shown around the central one, at different positions and distances from the main stimulus (Fig. 1). The task of the subject is to press a button on the mouse each time a peripheral stimulus is perceived.

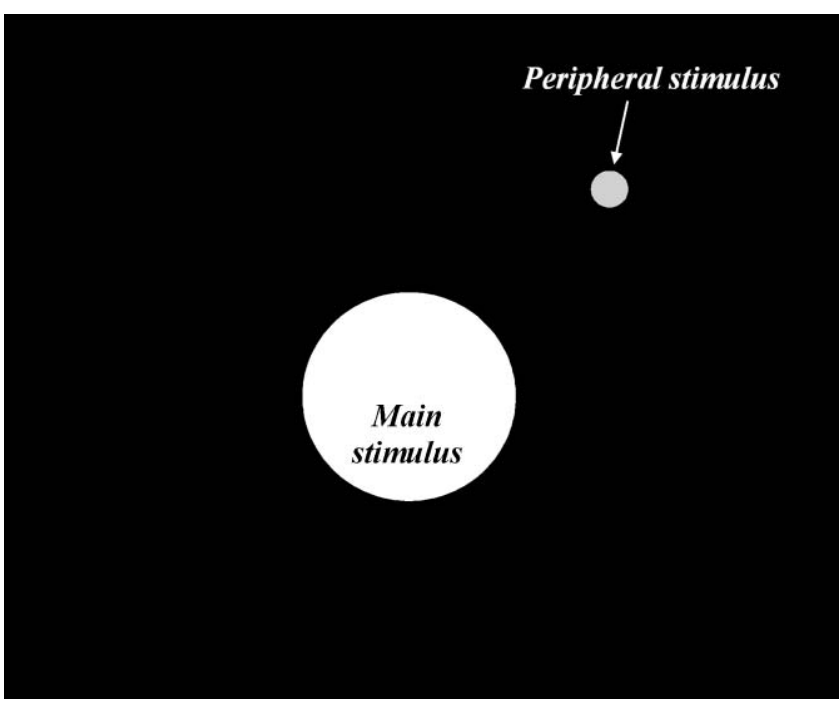

Fig. 1 Extended scheme of the test showing the main stimulus and one of the peripheral stimuli around it over the monitor background.

Before a session starts, the test can be setup with the following different variables:

1. Spatial variables (shown in pixels). At this point, the main-stimulus size as well as the peripheral-stimuli size can be selected. In conventional tests, the main stimulus is bigger and more luminous than the peripheral one to simulate a high-luminance stimulus that produces the visual disturbances perceived by the observer. These variables should be studied according to the experiment and type of patient. The size and luminance of the peripheral stimuli depend on the observer (the test parameters could differ for a young observer with keratitis versus an older patient with ARMD). The number of semiaxes also can be selected, as well as the number of stimuli per semiaxis (i.e., the number of peripheral stimuli distributed along each semiaxis of the configuration) and the maximum radius (the distance, in pixels, measured from the center of the main stimulus along a semiaxis to the center of the farthest peripheral stimulus along that semiaxis).

2. Temporal variables (shown in seconds). With the software, the darkness-adaptation time and the adaptation time to the luminance of the main stimulus can be set. In addition, regarding the peripheral stimuli, the time displayed on the monitor (exposure or on-period) and the time lapse between stimuli (refresh) can be selected. The refresh time presents a minimum and a maximum value to be set so that, for each peripheral stimulus, the refresh time takes a random value between the minimum and maximum to minimize false responses given by the observer. In the experiment, there is an option to select whether the main stimulus is always presented on the monitor (activated option) or only when the peripheral stimuli are shown (nonactivated option). It is advisable to activate the option, because showing the central stimulus helps maintain the fixation of the observer. 
An experimental session consists, sequentially, of darkness adaptation (to the luminance of the monitor background), an adaptation time to the main stimulus, and progressively, peripheral stimuli are shown randomly around the central one, the observer's task being to detect these stimuli. The information is stored, and when the test is finished, the software gives a result graph and parameter that quantifies the visual disturbances perceived by the observer.

\subsection{Visual-Disturbance Index}

The discrimination capacity of peripheral stimuli in the presence of visual disturbances (such as veils of stray light, glare, and halos generated by a central high-luminance stimulus) is evaluated by a parameter called the visual-disturbance index, or simply, disturbance index, as some authors have indicated. ${ }^{6}$ After the temporal and spatial parameters have been assigned and the test is finished, this index is calculated as a ratio between nondetected stimuli and all the peripheral stimuli presented to the observer [Eq. (1)]. This ratio takes into account the distance of each not-detected peripheral stimulus to the main-stimulus center, adjusted for the times that the corresponding peripheral stimulus is not detected by the subject. The distance dependence is also present in the denominator, where all the peripheral stimuli are considered. The disturbance index can be expressed in two ways: linear and quadratic (Halo software gives both indexes). In this work, we use the quadratic disturbance index $\rho_{q}$, as other studies have done, $, 6,7$ and it is simply called the disturbance index, which is calculated by

$$
\rho_{q}=\left(\sum_{i=1}^{N} p_{i} r_{i}^{2}\right) /\left(p \sum_{i=1}^{N} r_{i}^{2}\right),
$$

where $r_{i}$ is the distance (in pixels) from the center of the central stimulus to the center of the $i$-peripheral stimulus, considering a concrete semiaxis; $N$ is the total number of peripheral stimuli; $p$ is the total weight (number of times that each stimulus $i$ is shown), and once selected is the same for all the peripheral stimuli; and $p_{i}$ is the number of times over the total weight $\left(p_{i} \leq p\right)$ that the $i$-peripheral stimulus is not detected by the subject.

The disturbance index takes values of between 0 and 1 in such a way that the greater the index is, the lower the discrimination capacity. Therefore, the subject has more difficulty in detecting the peripheral stimuli, indicating a greater influence of visual disturbances, such as glare, a veil of stray light over the retinal image, or visual halos around luminous stimuli.

\subsection{Testing the Software}

\subsubsection{Patients}

We checked the software with two groups of patients differing in their ocular pathology: patients affected with keratitis and patients with ARMD, both pathologies with high prevalence. All patients were diagnosed by the same ophthalmologist in the University Clinic Hospital of Granada, Spain.

Keratitis is an important cause of infection-associated blindness and caused by a variety of pathogens, including viruses, fungi, bacteria, and amoeba. ${ }^{8,9}$ This disease damages the cornea because of intraocular inflammation, epithelial defects, ulcers, and corneal scrapes and scars. ${ }^{8,9}$ Patients with keratitis have diminished optical quality and a deteriorated visual performance. ${ }^{10,11}$ This study included 15 patients with unilateral keratitis with a mean age of $34.2 \pm 11.6$ years (standard deviation) and a range of 14-62 years. Patients with a Snellen visual acuity lower than $6 / 15$ or any other ocular diseases except for keratitis were excluded from the study. Monocular visual acuity was measured. In cases of ammetropes, visual acuity was measured with their best correction. Eight patients were emmetropes and seven were corrected ammetropes. The average refraction equivalent was $-0.5 \pm 1.5 \mathrm{D}$. Informed consent was given by each patient before the examination, in accordance with the Helsinki Declaration. Data on optical quality and visual performance for the patients with keratitis were taken before initiating treatment. In cases of herpetic keratitis, the treatment was acyclovir and steroids in topical administration, whereas in bacterial keratitis the therapy involved topical antibiotics, depending on organism sensitivities.

ARMD, in developed countries, is the leading cause of severe and central vision loss among the elderly. ${ }^{12}$ This pathology impairs macular function, causing reduced visual acuity and diminished contrast sensitivity. ${ }^{13,14}$ In the worst cases, it causes a complete loss of central vision, interfering with reading or performing tasks that require the ability to see in detail. ${ }^{15}$ Data were taken from 14 patients with unilateral ARMD, including ammetropes or patients with optimal correction, in different states of this retinal pathology. Subjects with a history or signs of other diseases relevant to visual function were excluded. The ages of ARMD patients ranged from 53 to 81 years $(70.9 \pm 7.4$ years), and the average refractive error was $-0.1 \pm 3.0 \mathrm{D}$. For all patients, we took data on Snellen visual acuity with their best correction. All participants in the experiments gave their informed consent in accordance with the Helsinki Declaration.

\subsubsection{Discrimination-capacity measurements}

For all keratitis patients, we selected the same values for the software parameters. The LCD-monitor resolution was set at $1024 \times 768$ pixels, and the distance from the observer position to the monitor was $2 \mathrm{~m}$. The size of the stimuli was 20 pixels for the radius of the main stimulus and 2 pixels for the radius of the peripheral stimuli, subtending 0.38 and $0.04 \mathrm{deg}$, respectively, as in other works. ${ }^{6,16}$ The luminance of the stimuli, measured by the spectroradiometer Spectra Scan PR-650 (Photo Research, Incorporated, Chatsworth, California), was of $171.9 \mathrm{~cd} / \mathrm{m}^{2}$ for the main stimulus and of $36.1 \mathrm{~cd} / \mathrm{m}^{2}$ for the peripheral one, with the luminance for the background monitor being $0.34 \mathrm{~cd} / \mathrm{m}^{2}$. The monitor showed a total of 36 peripheral stimuli around the central one, distributed along 12 semiaxes (three stimuli per semiaxis). The maximum radius of each semiaxis was 50 pixels (the most distant stimulus being 50 pixels from the center of the main stimulus). Previous results in our laboratory showed these spatial parameters to be suitable for performing the test in different groups of observers. This configuration agrees with spatial parameters used in other works. ${ }^{6,16}$

Because of advanced age and high deterioration level of vision, ARMD patients had difficulties in detecting peripheral stimuli with the keratitis-patient configuration for the peripheral stimuli. This aspect was checked in ARMD eyes, and in several 
cases, the results with this configuration showed the maximum value for the disturbance index (disturbance index $=1$ ), thereby losing information in the trend with the Strehl ratio. To achieve a true correlation in the parameters studied, we increased the size of the peripheral stimuli from 2 to 3 pixels, subtending $0.06 \mathrm{deg}$ from the observer's position. In this case, the maximum radius was 60 pixels, to compensate the increment in the peripheralstimuli size and to extend the region of the stimuli detection, allowing a complete evaluation of the visual disturbances despite the visual deterioration level in these subjects. The peripheralstimulus size used in ARMD patients was not suitable for the keratitis patients, because the majority of the keratitis patients were capable of detecting all of the three-pixel stimuli and, therefore, some cases would reach the lowest disturbance index in diseased eyes (disturbance index $=0$ ), truncating the graph for the patients with the highest Strehl ratios and thus masking the true correlation with the optical-quality parameter. This aspect is explained, because the aim of this work is basically to study the correlation between image quality and discrimination capacity for two different ocular pathologies, using a new visual test, without a detailed comparison between these pathologies. The rest of the parameters were equal for ARMD and keratitis patients. In all cases, we used a weight of $1(p=1)$, taking $p_{i}$ values of 0 (peripheral stimulus detected) or 1 (stimulus nondetected). Preliminary results revealed no great differences for the disturbance index in subjects who underwent the test with a weight of $p=1$ or with $p>1$, but we should indicate that a higher level of accuracy was attained with $p=3$, a typical value in other works. However, for the ARMD patients, due to aging effects that reduce their visual reaction time and the attention level, the exposure to peripheral stimulus was slightly increased from 0.8 to $1.2 \mathrm{~s}$, as well as the refresh time, making the test time longer. For a $p>1$ configuration, the test would be much longer and too tiring for the ARMD patients (for $p=3$, the testexecution time would be practically tripled), as some subjects reported in trials prior to the session. For this reason, we used a $p=1$ configuration. To maintain similar experimental conditions, we used the same temporal and weight configurations, varying neither luminance of the main stimulus nor that of the peripheral stimuli for the two groups of patients.

The measurements were performed with the minimum background luminance of the monitor to provide high contrast with the luminous stimulus. A session was performed as follows: after a 3-min adaptation period to darkness of the monitor background, ${ }^{17,18}$ there was a 1 -min adaptation to the main stimulus (a high-luminance stimulus), and the observer was randomly presented with peripheral stimuli around the central stimulus. Previously, the observer position was fixed in front of the monitor with a chin and forehead rest, and an initial trial test was executed to check the correct understanding of the test. The patient, on detecting peripheral spots, pressed a button on the mouse, storing this information for subsequent treatment and calculation of the disturbance index.

\subsubsection{Optical-quality measurements}

To check the existence of a correlation between optical quality, as an objective measurement, and the discrimination capacity as visual function to evaluate visual performance, we also took data from a visual-quality device, the Optical Quality Analysis
System [(OQAS), Visiometrics SL, Tarrasa, Spain], based on the double-pass technique. ${ }^{1}$ In this device, a point source (an infrared laser diode, $\lambda=780 \mathrm{~nm}$ ) is projected onto the retina. The reflected light passes through ocular media, and the double-pass image is recorded by a camera. ${ }^{1}$ This device provides data on diffraction, ocular aberrations, scattering, and retinal reflection that affects the retinal-image quality, reducing visual quality of the subject. These objective optical devices are useful not only in clinical applications, such as refractive and cataract surgery, where the influence of scattering could be great, but also in patients with some ocular pathology, as studied here, where a deterioration of the ocular media increases aberrations, intraocular scattering, and retinal reflection, resulting in a worse retinal-image quality. ${ }^{10,11}$ The device includes a motorized optometer to correct the patient's defocus. ${ }^{1}$ OQAS measurements were made for a 5 -mm pupil in keratitis patients and, due to an advanced age, for a 4-mm pupil in ARMD patients, because most of them did not reach a 5-mm pupil.

For a quantitative measurement of the visual quality, we took the Strehl ratio, a parameter commonly used for estimating overall optical quality ${ }^{1,2}$ defined as the ratio between the modulation transfer function (MTF) area of the eye and the diffraction-limited MTF area. The MTF represents the contrast loss resulting from the ocular optics on a sinusoidal grating as a function of its spatial frequency. The Strehl ratio ranges from 0 to 1 . A lower value of this parameter indicates that there is a greater contribution of the aberrations and ocular scattering and therefore poorer optical quality. More information on this device can be found in other papers. ${ }^{1,2}$

Statistical analysis used in this work was performed using a mean-comparison test to detect significant differences between pathological and healthy fellow eyes. For the $p$-value, the condition $p<0.05$ was considered to be statistically significant.

\section{Results}

We checked a new visual test based on simple software for testing the discrimination capacity under low-illumination conditions in two groups of patients corresponding to two different ocular pathologies: ARMD and keratitis. The results from the software were compared to objective data provided by the double-pass device. Table 1 shows the average data (standard deviation) for the Strehl ratio, found with the OQAS, and the disturbance index, provided by our Halo software, in patients with one of the two ocular pathologies, differentiating between eyes with disease and contralateral healthy eyes.

For keratitis eyes, the Strehl ratio ranged from 0.075 to 0.240 , with a mean value of $0.17 \pm 0.05$ (standard deviation). In the case of healthy fellow eyes, the mean was $0.29 \pm 0.14$, indicating a significantly lower value $(p=0.008)$ in the Strehl ratio for keratitis eyes, and therefore, a stronger influence of aberrations, retinal reflection, and intraocular scattering, as some works have shown. ${ }^{10,11}$ In accordance with previous research, ${ }^{10}$ the same tendency was found in ARMD patients where the average Strehl ratio was $0.10 \pm 0.03$ for pathological eyes (ranging from 0.056 to 0.156 ) and $0.18 \pm 0.05$ for contralateral healthy eyes, the differences being significantly lower for the diseased eyes ( $p=0.012$ ). Analyzing the averages, we found the mean value for the Strehl ratio to be significantly lower in keratitis and ARMD eyes compared to the mean for the healthy fellow 
Castro et al.: New testing software for quantifying discrimination capacity ...

Table 1 Average data for the Strehl ratio and disturbance index. Standard deviation included.

\begin{tabular}{llllll} 
& \multicolumn{2}{c}{ ARMD } & & \multicolumn{2}{c}{ Keratitis } \\
\cline { 2 - 3 } & Strehl ratio & Disturbance index & & Strehl ratio & Disturbance index \\
\hline Average for eyes with disease & $0.10 \pm 0.03$ & $0.57 \pm 0.11$ & & $0.17 \pm 0.05$ & $0.67 \pm 0.10$ \\
Average for contralateral healthy eyes & $0.18 \pm 0.05$ & $0.45 \pm 0.10$ & & $0.29 \pm 0.14$ & $0.44 \pm 0.09$ \\
Mean comparison ( $p$-value) & 0.012 & 0.021 & 0.008 & 0.011 \\
\hline
\end{tabular}

eyes. Regarding the discrimination capacity, in keratitis eyes the disturbance index ranged from 0.54 to 0.84 , with a mean value of $0.67 \pm 0.10$, whereas for the contralateral healthy eyes, the mean was $0.44 \pm 0.09$ (the mean was significantly higher, $p=0.011$, in diseased eyes). In ARMD eyes, the disturbance index took values from 0.40 to 0.72 , with a mean value of $0.57 \pm 0.11$, significantly higher $(p=0.021)$ than the mean for the healthy fellow eyes, $0.45 \pm 0.10$. These results indicate that the disturbance index was significantly higher in pathological eyes compared to healthy fellow eyes.

For each patient the disturbance index was higher for the diseased eye in $86.7 \%$ of the keratitis patients (13 of the 15 keratitis patients) and $78.6 \%$ in the ARMD patients (11 of 14 ARMD patients), with the differences in that parameter being significant (for the average $p=0.021$ ). The higher disturbance index indicates a deteriorated discrimination capacity resulting from the ocular pathology. For the retinal-image quality, we found similar results: in almost all subjects $(>78 \%)$, the Strehl ratio was lower in the pathological eye, indicating worse retinal-image quality due to a combination of greater intraocular scattering, aberrations, and retinal reflection.

Figure 2 presents the results for the disturbance index in ARMD eyes (4-mm pupil) as a function of the Strehl ratio. We found a significant descending correlation $(r=0.85, p<0.05)$ between parameters studied. For keratitis eyes (5-mm pupil), Fig. 3 shows the results for the disturbance index as a function of the Strehl ratio, revealing a significant descending correlation

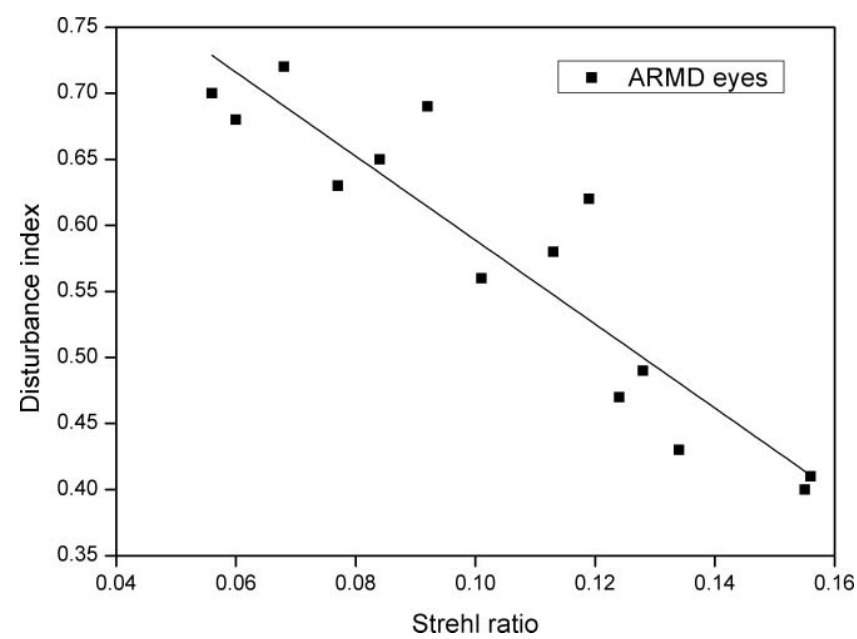

Fig. 2 Disturbance index as a function of the Strehl ratio for the eye with disease in ARMD patients. $(r=0.81, p<0.05)$. In both types of pathological eyes, the lower the Strehl ratio was, the higher the disturbance index was, and therefore, the lower the discrimination capacity was for peripheral stimuli, indicating a higher influence of different visual disturbances perceived by the observer, due to the ocular pathology (such as glare sensation, a veil of stray light, and visual halos) becoming an important influence on visual performance.

We found that the lowest Strehl-ratio values were for the ARMD eyes due to the high level of visual deterioration in these eyes (Fig. 2). Although optical quality data were referred to a different pupil size ( $4 \mathrm{~mm}$ for ARMD and $5 \mathrm{~mm}$ for keratitis patients), a lower Strehl ratio is expected for a 5-mm pupil in ARMD patients, because ocular aberrations and intraocular scattering increase for a higher pupil size. Other results have shown optical quality in ARMD and keratitis patients (taking into account the Strehl ratio and higher-order aberrations), resulting in worse optical quality for the ARMD eyes compared to the keratitis eyes. ${ }^{10}$ In the visual-disturbances test performed here, a different spatial configuration was used for the two groups of observers (peripheral-stimuli size and maximum radius were increased for ARMD patients), which did not allow us a quantitative comparison between these ocular pathologies. Using the keratitis-patient setup in the software, the discrimination capacity in ARMD patients studied here was intuitively lower compared to keratitis patients, because in some cases, the maximum value of 1 for the disturbance index was reached, and for this reason, we increased the size of the peripheral stimuli.

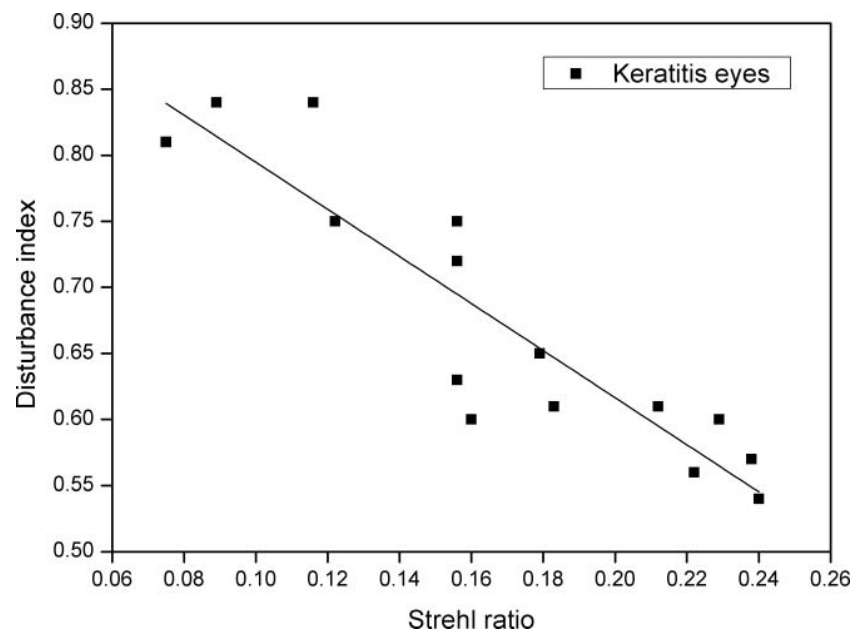

Fig. 3 Disturbance index as a function of the Strehl ratio for the diseased eye in keratitis patients. 
The experimental setup in the visual-disturbance test performed here should be adapted to each ocular pathology or group of observers, because this would allow us to use the test in a wide range of experimental conditions (patients with different ocular pathologies, those having undergone refractive surgery, those operated on for cataract surgery, etc.), offering an important advantage of the test. In ARMD eyes, a lower Strehl ratio strongly increased the disturbance index compared to the trend in keratitis eyes (for a linear regression, the slope of the line adjusted to the keratitis-eyes data was $-1.78 \pm 0.23$, and for the ARMD-eyes data $-3.18 \pm 0.38$ ). Although numerical values of the disturbance index are noncomparable between ARMD and keratitis patients, these trends with the optical quality suggest a higher deterioration level of visual performance in ARMD patients (a stronger slope) compared to keratitis patients, as other results have shown for contrast-sensitivity function. ${ }^{10}$

\section{Discussion}

According to our results, ARMD and keratitis patients showed a lower Strehl ratio and higher disturbance index in the eye with disease than in the contralateral healthy eye, reflecting a deterioration of the retinal-image quality and a lower discrimination capacity. Both pathologies directly affect the functionality of the eye. In keratitis patients, the functionality is affected due to corneal inflammation, epithelial defects, ulcers, and corneal scrapes and scars. Therefore, the transparency of the cornea is altered in particular regions, and consequently, scattered light and aberrations increase, resulting in poor retinal-image quality (a lower Strehl ratio). When a high-luminance stimulus is seen by a keratitis patient, the level of scattered light and aberrations increase due to these corneal alterations, provoking visual disturbances perceived by the patient (glare, veil of stray light, or halos around the main luminous stimulus).

On the other hand, the reason that ARMD eyes showed a lower Strehl ratio with respect the contralateral healthy eye could be abnormalities in the state of the retina (e.g., the retinal pigment epithelium) that increase the amount of scattered light after reflection onto it, deteriorating the retinal-image quality and resulting in the lower Strehl ratio. ${ }^{10,12}$ This scattered light contributes to the glare sensation and the veil of stray light over the retinal image, as well as to the halos perceived by the patient around central lights, decreasing the discrimination capacity to peripheral stimuli, and consequently, increasing the disturbance index, as demonstrated in our work.

In summary, scattered light through the eye, reflected light on the retina, and ocular aberrations caused by the alterations indicated contribute to the glare sensation and other visual alterations in the pathological eye, diminishing the discrimination capacity, and ultimately deteriorating the visual performance of the subject. These interactions of light passing through the ocular media, including the reflection on the retina, all together influence retinal-image quality, as demonstrated in our work, ${ }^{19,20}$ revealing as well a significant ascending correlation between discrimination capacity and retinal-image quality. Therefore, our Halo software test is useful because the most used visual tests, such as visual acuity, are not adequate for a complete evaluation of visual performance in patients with such ocular pathologies as the ARMD or keratitis patients studied here, and visual performance would not be completely characterized, omitting visual functions as important as the discrimination capacity. In the presence of visual disturbances, as generated by the ocular pathologies studied here, there is a reduction in the discrimination capacity to peripheral stimuli around the high-luminance stimulus, deteriorating visual performance. Therefore, the vision examination could be completed with the study of the subject's discrimination capacity. This capacity is key, for example, in daily tasks such as reading or writing, as well as in night driving, where not detecting peripheral stimuli around headlights of the oncoming traffic (e.g., pedestrians crossing the street) could lead to a traffic accident. Our results agree with other works that have shown deteriorated visual performance in patients with such ocular pathologies as ARMD or keratitis, reflecting a reduction in functions such as the contrast-sensitivity function ${ }^{10,11}$ but also in the discrimination capacity studied in our work.

The use of a simple psychophysical test as developed here allows us not only to evaluate an important aspect of vision, the discrimination capacity (by means of the disturbance index), but also to characterize the visual performance of that subject, this becoming an important tool for the study and time course of different ocular pathologies that can develop asymptomatically to advanced stages. We believe that early diagnosis could also be a potential application of this software. A noteworthy point is that the results for ARMD and keratitis patients could be extended to other ocular pathologies, making the Halo software a versatile tool in different types of patients. Furthermore, this software could be useful in cataract and refractive-surgery patients ${ }^{6,7}$ who experience night-vision disturbances, such as starbursts and halos around lights, which reduce the capacity to discriminate peripheral luminous stimuli around a high-luminance stimulus, as other works have shown by the use of a halometer. ${ }^{6,7}$

In this work, we should indicate certain limitations, because the optical quality of the eye was measured with infrared light $(\lambda=780 \mathrm{~nm})$, whereas the discrimination-capacity tests were performed with white light. Infrared light is used because it is more comfortable for the patient and provides adequate estimates of the retinal image quality compared to visible light. ${ }^{10}$ Intraocular scattering and eye aberrations contribute to optical quality, affecting the Strehl-ratio values. On the one hand, eye scattering depends on the wavelength of the radiation ${ }^{21}$ and the Strehl ratio values could differ if data are taken for a different wavelength. On the other hand, with respect to ocular aberrations, some results have reported that there are no great variations in the monochromatic aberrations with wavelength, ${ }^{22}$ and the effect of correcting chromatic aberration for psychophysical measurements, such as the contrast sensitivity function, is minor. $^{23}$

In conclusion, the quantification of visual disturbances from an ocular pathology is a key aspect to take into account when evaluating visual performance, time course, and monitoring of pathology, and it is an aspect that could be overlooked by other tests. Therefore, we have developed simple software to evaluate these disturbances by means of calculating the disturbance index, not only in refractive surgery or cataract patients ${ }^{6,16}$ where night-vision disturbances (glare, halos, and starbursts) are present, but also in patients with some ocular pathologies, such as ARMD or keratitis, where visual performance is deteriorated. Another important task performed by the Halo software is quantification, being more accurate than some questionnaires used to evaluate qualitatively visual disturbances or nocturnal 
symptoms in visual tasks where results are limited to glare sensation, veils of stray light, halos, and starbursts reported by the subjects. Therefore, to quantify visual disturbances by means of the discrimination capacity and to evaluate visual performance, the Halo software test is a powerful tool, being a reliable method, easy to perform, and available to any examiner. The effectiveness of the Halo software is reaffirmed by a significant descending correlation between the retinal-image quality and the visual disturbances perceived by the subject.

\section{Acknowledgments}

We thank David Nesbitt for translating the text into English. This research was supported by the Ministerio de Educación y Ciencia (Spain) Grant No. FIS2009-07482 and Junta de Andalucía (Spain) Grant No. P06-FQM-01359.

\section{References}

1. F. Diaz-Douton, A. Benito, J. Pujol, M. Arjona, J. L. Guell, and P. Artal, "Comparison of the retinal image quality with a Hartmann-Shack wavefront sensor and a double-pass instrument," Invest. Ophthalmol. Vis. Sci. 47(4), 1710-1716 (2006).

2. J. L. Guell, J. Pujol, M. Arjona, F. Diaz-Douton, and P. Artal, "Optical quality analysis system: instrument for objective clinical evaluation of ocular optical quality," J. Cataract Refract. Surg. 30(7), 1598-1599 (2004).

3. J. Z. Liang, B. Grimm, S. Goelz, and J. F. Bille, "Objective measurement of wave aberrations of the human eye with the use of a Hartmann-Shack wavefront sensor," J. Opt. Soc. Am. A 11(7), 1949-1957 (1994).

4. J. S. McLellan, P. M. Prieto, S. Marcos, and S. A. Burns, "Effects of interactions among wave aberrations on optical image quality," Vis. Res. 46(18), 3009-3016 (2006).

5. J. Buhren, T. Martin, A. Kuhne, and T. Kohnen, "Correlation of aberrometry, contrast sensitivity, and subjective symptoms with quality of vision after LASIK," J. Refract. Surg. 25(7), 559-568 (2009).

6. R. Gutierrez, J. R. Jimenez, C. Villa, J. A. Valverde, and R. C. Anera, "Simple device for quantifying the influence of halos after lasik surgery," J. Biomed. Opt. 8(4), 663-667 (2003).

7. J. R. Jimenez, C. Ortiz, E. Hita, and M. Soler, "Correlation between image quality and visual performance," J. Mod. Opt. 55(4-5), 783-790 (2008).

8. P. Y. Robert, A. Liekfeld, S. Metzner, S. Ranger-Rogez, J. P. Adenis, F. Denis, C. Hartmann, and U. Pleyer, "Specific antibody production in herpes keratitis: intraocular inflammation and corneal neovascularisation as predicting factors," Graefes Arch. Clin. Exp. Ophthalmol. 244(2), 210-215 (2006).
9. J. P. Whitcher and M. P. Srinivasan, "Corneal ulceration in the developing world - a silent epidemic," Br. J. Ophthalmol. 81(8), 622-623 (1997).

10. J. R. Jimenez, C. Ortiz, F. Perez-Ocon, and J. J. Castro, "Objective and subjective optical-quality measurements in subjects with keratitis and age-related macular degeneration," J. Mod. Opt. 55(15), 2371-2380 (2008).

11. J. R. Jimenez, C. Ortiz, F. Perez-Ocon, and R. Jimenez, "Optical image quality and visual performance for patients with keratitis," Cornea 28(7), 783-788 (2009).

12. A. D. Kulkarni and B. D. Kuppermann, "Wet age-related macular degeneration," Adv. Drug Del. Rev. 57(14), 1994-2009 (2005).

13. M. F. Alexander, M. G. Maguire, T. M. Lietman, J. R. Snyder, M. J. Elman, and S. L. Fine, "Assessment of visual function in patients with age-related macular degeneration and low visual-acuity," Arch. Ophthalmology 106(11), 1543-1547 (1988).

14. P. J. Mackenzie, T. S. Chang, I. U. Scott, M. Linder, D. Hay, W. J. Feuer, and K. Chambers, "Assessment of vision-related function in patients with age-related macular degeneration," Ophthalmology 109(4), 720 729 (2002).

15. N. Bansback, C. Czoski-Murray, J. Carlton, G. Lewis, L. Hughes, M. Espallargues, C. Brand, and J. Brazier, "Determinants of health related quality of life and health state utility in patients with age related macular degeneration: the association of contrast sensitivity and visual acuity," Qual. Life Res. 16(3), 533-543 (2007).

16. J. R. Jimenez, C. Villa, R. G. Anera, R. Gutierrez, and L. J. del Barco, "Binocular visual performance after LASIK," J. Refract. Surg. 22(7), 679-688 (2006).

17. J. R. Jimenez, M. Rubino, J. A. Diaz, E. Hita, and L. J. Del Barco, "Changes in stereoscopic depth perception caused by decentration of spectacle lenses," Optom. Vis. Sci. 77(8), 421-427 (2000).

18. J. R. Jimenez, J. L. Olivares, F. Perez-Ocon, and L. J. Del Barco, "Associated phoria in relation to stereopsis with random-dot stereograms," Optom. Vis. Sci. 77(1), 47-50 (2000).

19. P. Artal, M. Ferro, I. Miranda, and R. Navarro, "Effects of aging in retinal image quality," J. Opt. Soc. Am. A 10(7), 1656-1662 (1993).

20. J. J. Castro, J. R. Jimenez, E. Hita, and C. Ortiz, "Influence of interocular differences in the Strehl ratio on binocular summation," Ophthalm. Physiol. Opt. 29(3), 370-374 (2009).

21. N. Lopez-Gil and P. Artal. "Comparison of double-pass estimates of the retinal-image quality obtained with green and near-infrared light," J. Opt. Soc. Am. A 14(5), 961-971 (1997).

22. L. Llorente, L. Diaz-Santana, D. Lara-Saucedo, and S. Marcos, "Aberrations of the human eye in visible and near infrared illumination," Optom. Vis. Sci. 80(1), 26-35 (2003).

23. G. Y. Yoon and D. R. Williams, "Visual performance after correcting the monochromatic and chromatic aberrations of the eye," J. Opt. Soc. Am. A 19(2), 266-275 (2002). 